\title{
Evolution of Magnetic Resonance Imaging Changes Associated with Cerebral Hypoxia-Ischemia and a Relatively Selective White Matter Injury in Neonatal Rats
}

\author{
SHUZHEN MENG, MIN QIAO, KATHRYN SCOBIE, BOGUSLAW TOMANEK, AND URSULA I. TUOR \\ Institute for Biodiagnostics (West) [S.M., M.Q., KS, B.T., U.I.T.], National Research Council of Canada, Calgary, Alberta, Canada T2N \\ 4N1; Department of Pediatrics [S.M.], China Medical University, The Second Clinical College, Shenyang, Liaoning, China 110004; \\ Experimental Imaging Centre [B.T., U.I.T], University of Calgary, Faculty of Medicine, Alberta, Canada T2N 4N1
}

\begin{abstract}
We hypothesized that a combination of quantitative magnetic resonance imaging (MRI) sequences would detect a differential evolution of hypoxic-ischemic changes in white matter compared with gray matter in a recently developed model of unilateral mild cerebral hypoxia-ischemia in the 7-d-old rat. Using this model, which involved unilateral carotid artery occlusion and exposure to hypoxia for 45-50 min, maps of apparent diffusion coefficients of water $(\mathrm{ADC}), \mathrm{T}_{1}, \mathrm{~T}_{2}$, and cerebral blood flow (CBF) were acquired either before hypoxia-ischemia or at 1,24 , or $48 \mathrm{~h}$ and at $7 \mathrm{~d}$ post-hypoxia-ischemia followed by brain processing for histology. At $1 \mathrm{~h}$ post-hypoxia-ischemia, MRI changes in white matter ipsilateral to the hypoxia-ischemia were not as pronounced as those in gray matter. However, increases in $\mathrm{T}_{1}, \mathrm{~T}_{2}$ and $\mathrm{ADC}$ and decreases in $\mathrm{CBF}$ within white matter enhanced over time, with changes being maximal at $48 \mathrm{~h}$ post-hypoxia-ischemia, whereas changes in the cortical gray matter normalized over this time. By $7 \mathrm{~d}$ post-hypoxia-ischemia, there were no differences in $\mathrm{ADC}, \mathrm{T}_{1}, \mathrm{~T}_{2}$, or CBF between hemispheres despite there being histologic changes in white matter within the hypoxic-ischemic hemisphere including increased glial proliferation and reactivity, reduced myelin basic protein, and increased cell death. The results demonstrate that increases in ADC and $\mathrm{T}_{2}$ observed subacutely in the days following hypoxia-ischemia are associated with rather selective white matter damage and suggest that diffuse white matter hyperintensities and increased ADC reported in infants are transient MRI changes post- hypoxia-ischemia. (Pediatr Res 59: 554-559, 2006)
\end{abstract}

$\mathrm{I}^{\mathrm{n}}$ mprovements in neonatal medicine have been accompanied by an increase in the survival of small premature infants, with substantial numbers having adverse neurologic sequelae related to cerebral white matter injury (1-3). Although the pathogenesis may be uncertain, such brain injury can be a result of hypoxic-ischemic damage to the white matter consisting of focal cystic lesions deep in the periventricular white matter or a more diffuse noncystic white matter injury. Diagnosis of such white matter injury at an early acute stage is

Received July 19, 2005; accepted November 28, 2005.

Correspondence: Ursula Tuor, Ph.D., Institute for Biodiagnostics (West), National Research Council, 3330 Hospital Drive, NW, Calgary, Alberta, Canada T2N 4N1; e-mail: Ursula.Tuor@nrc.ca

This study was supported by grants from the Robertson Fund for Cerebral Palsy and the Heart and Stroke Foundation of Alberta.

DOI: $10.1203 / 01 . p d r .0000203099 .40643 .84$ necessary for developing optimal clinical management and treatment strategies.

Despite MRI techniques gaining acceptance for their evaluation of cerebral hypoxic-ischemic injury in neonates, information on the acute hypoxic-ischemic MRI changes and their evolution in immature white matter compared with gray matter remains limited (3-8). In general, MRI studies have shown that diffusion weighted imaging (DWI) is able to detect acute changes in the cerebral white matter of preterm infants where these changes are often not demonstrated on conventional MRI, i.e. $\mathrm{T}_{1}$ or $\mathrm{T}_{2}(5,9)$. In addition, more recent studies in term infants with hypoxic-ischemic encephalopathy show that the ADC of water is decreased in severely damaged white matter within the first week after birth and then tends to increase after the first week $(7,8,10)$.

Our recent study in neonatal rats has demonstrated that selective white matter injury can be induced by a mild hypoxic-ischemic insult and this rather selective white matter injury could be detected with $\mathrm{T}_{1}$ and $\mathrm{T}_{2}$ weighted imaging at $24 \mathrm{~h}$ following a hypoxic-ischemic insult (11). The onset, progression, and permanence of the hypoxic-ischemic white matter injury and how well it can be detected using MRI at time points other than $24 \mathrm{~h}$ following the insult are unknown. Different MRI sequences can provide different information concerning the underlying tissue proton changes, including whether there is increased tissue water (e.g. increased $\left.\mathrm{T}_{1}\right)$, cell swelling (e.g. increased ADC of water), or edema with bloodbrain barrier dysfunction and extravasation of proteins (e.g. increased $\mathrm{T}_{2}$ ). Thus, it is possible that a combination of imaging sequences including $\mathrm{T}_{1}, \mathrm{~T}_{2}, \mathrm{ADC}$, and cerebral perfusion imaging would provide additional diagnostic or pathophysiological information relevant to the evolution of the insult. We hypothesized that there would be a distinctive progression of tissue changes in white matter in the hours and days following a rather mild hypoxic-ischemic insult where

Abbreviations: ADC, apparent diffusion coefficient; CBF, cerebral blood flow; GFAP, glial fibrillary acidic protein; MBP, myelin basic protein; TUNEL, terminal deoxynucleotidyl transferase-mediated biotinylated dUTP nick end labeling 
some combination of rather standard quantitative MRI methods would be optimal for their noninvasive detection. Thus, in the present study, changes in $\mathrm{ADC}, \mathrm{T}_{1}, \mathrm{~T}_{2}$, and $\mathrm{CBF}$ were measured in cerebral white and gray matter at several different time points following a mild hypoxic-ischemic insult in 7-dold rats.

\section{MATERIALS AND METHODS}

Model of cerebral hypoxic-ischemic white matter injury. Experiments were performed according to Canadian Council on Animal Care guidelines using protocols approved by the NRC and local Animal Care Committees. Pregnant Wistar rats (Charles River Laboratories, Montreal, Quebec, Canada) gave birth and their litters were culled to nine to 10 pups. Twenty-nine rats at the age of $7 \mathrm{~d}$ were subjected to a sham procedure $(n=10)$ or a cerebral hypoxic-ischemic insult $(n=19)$ expected to produce a relatively selective white matter injury as described previously (11). Briefly, the right carotid artery was ligated and severed under $2.0-2.5 \%$ isoflurane anesthesia. The incision site was infiltrated with bupivacaine and closed. After $1-2 \mathrm{~h}$ of recovery, rats were exposed to hypoxia (humidified $8 \%$ oxygen in nitrogen) for $45-50 \mathrm{~min}$. Chamber temperature was maintained at $34.5 \pm 0.1^{\circ} \mathrm{C}$ (body temperature of $36-37^{\circ} \mathrm{C}$ ) during hypoxia using a neonatal incubator and heating lamp. In the sham control group, the right carotid artery was simply isolated and there was no exposure to hypoxia.

MRI. Rats were imaged at either $1(n=6), 24(n=7)$ or $48(n=6) \mathrm{h}$ after hypoxia-ischemia to follow changes that are known to be maximal in the first few days following stroke in the rat $(12,13)$. To examine more subacute changes, all animals were imaged again at $7 \mathrm{~d}$ after hypoxia-ischemia except for two with poor recovery that were lost to further study $(n=17)$. Control animals were imaged at either $8 \mathrm{~d}(n=5)$ or $2 \mathrm{wk}(n=5)$ of age. All images were acquired using a quadrature volume coil in a temperature-controlled chamber and an MRI system equipped with a 9.4-T/21-cm horizontal bore magnet (Magnex) and an Avance console (Bruker, Germany). The head was restrained with foam while the electrocardiogram and/or respiration were monitored. Images were acquired with a field of view of $2.5 \mathrm{~cm}^{2}$ and a data matrix of $256 \times 128$ for five slices $1.25 \mathrm{~mm}$ thick through the cerebrum. An ADC map was calculated from sets of diffusion weighted spin-echo images acquired using a repetition time (TR) of $1200 \mathrm{~ms}$, an echo time (TE) of $40 \mathrm{~ms}$, and $\mathrm{b}$ values of 57 and $1195 \mathrm{~s} / \mathrm{mm}^{2}$ that were acquired in $8 \mathrm{~min}$. $\mathrm{T}_{2}$ maps were generated using a monoexponential fit and 21 echoes collected using a spin-echo multislice imaging sequence acquired in $5 \mathrm{~min}(\mathrm{TR}=2500 \mathrm{~ms}$, $\mathrm{TE}=10 \mathrm{~ms}) . \mathrm{T}_{1}$ maps were calculated within a single posterior coronal slice from a series of eight inversion-recovery $\mathrm{T}_{1}$-weighted Snapshot-FLASH images acquired in $5 \mathrm{~min}(\mathrm{TR}=3.55 \mathrm{~ms}, \mathrm{TE}=2.1 \mathrm{~ms}$, increasing time of inversion delays of $234,503,831,1233,1751,2480,3728,9226 \mathrm{~ms})$. Perfusion imaging was performed in the same slice using an arterial spin labeling technique $(14)(\mathrm{TR} / \mathrm{TE}=3.55 / 2.1 \mathrm{~ms}$, flip angle $=12$ degrees, average of 32) with an adiabatic inversion pulse in a $1.5-\mathrm{G} / \mathrm{cm}$ field gradient followed by a TurboFLASH imaging sequence requiring the acquisition of a set of four images (two control and two inversion labeled) in $8 \mathrm{~min}$. CBF (in $\mathrm{mL} / \mathrm{g} / \mathrm{s})$ was calculated as $\mathrm{CBF}=\lambda\left(1 / \mathrm{T}_{1}+\delta\right)($ Mbcon - Mbinv $) / 2 \alpha \mathrm{Mbcon}$, using $\lambda$, the blood-brain barrier partition coefficient, as $0.9, \delta$ as $0.039 \mathrm{~s}^{-1}$, and $\alpha$ as 0.75 . Measured values included $T_{1}$ of brain; Mbcon, the intensity within control images; Mbinv, the intensity within images after arterial inversion of blood in the common carotid artery. After the final scan, animals were anesthetized with pentobarbital and then perfused transcardially with $4 \%$ paraformaldehyde. The brains were removed and cut into two parts at approximately $2 \mathrm{~mm}$ posterior to the bregma. They were further fixed in $4 \%$ paraformaldehyde and subsequently embedded in paraffin. Coronal sections $(7 \mu \mathrm{m})$ were cut at levels corresponding to posterior and anterior MRI slices.

MRI parameters were measured ipsilaterally and contralaterally within anterior or posterior slices located at approximately 0 and $5 \mathrm{~mm}$ from the bregma, respectively. Regions of interest consisted of white matter within the external capsule and the gray matter of parietal cortex and thalamus (posterior slice) or striatum (anterior slice). Images with movement artifacts were excluded. Data are presented as mean $\pm \mathrm{SD}$. Comparison of differences in means at different time points was performed using analysis of variance (ANOVA) and a post hoc Bonferroni $t$ test. Because of developmental changes over time, left-right differences between hemispheres at each time point were also compared using a paired $t$ test. Differences were considered significant at $p<0.05$.

Histologic staining. At least two adjacent slides were stained for anterior and posterior blocks for each staining method that included either standard staining with hematoxylin and eosin or immunohistochemically for myelin basic protein (MBP) or glial fibrillary acidic protein (GFAP). For immunohistochemistry, the slides were processed to remove paraffin, immersed in $3 \%$ hydrogen peroxide in methanol for 10 min followed by blocking with $10 \%$ normal goat serum (Jackson ImmunoResearch, West Grove, PA). The slides were then incubated with primary antibody to MBP (rabbit polyclonal antiMBP, Chemicon Int., Temecula, CA; 1:2000) or GFAP (rabbit polyclonal anti-GFAP, Chemicon Int.; $1: 2500$ ) overnight at $4^{\circ} \mathrm{C}$. This was followed by incubation with Bio-SP-conjugated affinity purified goat anti-rabbit $\operatorname{IgG}$ (Jackson ImmunoResearch; 1:200) for $1 \mathrm{~h}$ and streptavidin-horseradish peroxidase (Dako, Mississauga, Ontario, Canada; 1:400) for $30 \mathrm{~min}$ at room temperature and subsequent visualization using diaminobenzidine (Sigma Chemical Co., Oakville, Ontario, Canada) as a chromogen. Control slides incubated with blocking serum and without primary antibodies stained negative.

Terminal deoxynucleotidyl transferase-mediated biotinylated dUTP nick end labeling (TUNEL) was performed with an ApopTag ${ }^{\mathrm{R}}$ Red In Situ Apoptosis Detection Kit (Chemicon Int.) according to manufacturer's directions in anterior blocks. Negative controls were performed without active terminal deoxynucleotidyl transferase but including proteinase $\mathrm{K}$ digestion to control for nonspecific incorporation of nucleotides or for nonspecific binding of enzyme conjugate.

Changes in staining were analyzed semiquantitatively within regions corresponding to those in which quantitative MRI measures were made. Three fields in each region were assessed per section using light microscopy under $\times 40$ objective. GFAP staining was graded as mild, moderate, or severe for $<30,30-50$, or $>50$ positive reactive astrocytes per field, respectively, and TUNEL labeling was graded as normal, mild, moderate, or severe for $0-1$, $1-5,5-10$, or $10-20$ positive cells per field, respectively. The intensity of MBP immunoreactivity was estimated by measuring the relative optical density (OD) within the external capsule using an image analysis system and results were expressed as a ratio of ipsilateral/contralateral values.

\section{RESULTS}

To identify MRI changes with relatively selective white matter injury, we excluded animals that had substantial cortical and subcortical damage. Thus, of the 17 animals imaged and processed for histology, three were excluded from further analysis because of the appearance of cortical infarcts in hematoxylin and eosin-stained sections similar to that observed with a more severe 1.5 -h hypoxic-ischemic insult previously (11).

MRI. MRI changes compared between groups over time demonstrated differences influenced to a large extent by ontogenic changes in $\mathrm{T}_{1}, \mathrm{~T}_{2}$, and $\mathrm{ADC}$. Thus, when inspecting images (Fig. 1) and assessing quantitatively the effects of hypoxia-ischemia (Fig. 2), we focused on hypoxic-ischemic effects producing differences in ipsilateral and contralateral hemispheres rather than the differences observed between animals imaged at 7-9 d of age after hypoxia-ischemia and those at 2 wk of age.

Differences between hemispheres at an early time $(1 \mathrm{~h})$ after hypoxia-ischemia, resulted in modest ipsilateral increases in $\mathrm{T}_{1}$ and $\mathrm{T}_{2}$ and decreases in ADC and perfusion within gray matter of the posterior parietal cortex. The thalamus had similar changes in $\mathrm{T}_{1}$ and perfusion, whereas at this time, the only significant left-right difference within the white matter (external capsule) was an increase in $\mathrm{T}_{1}$. At 24 and $48 \mathrm{~h}$ after hypoxia-ischemia, there were no significant ipsilateralcontralateral differences in parietal cortex, whereas white matter ipsilaterally tended to have increases in $T_{1}, T_{2}$, and $\mathrm{ADC}$ and decreases in $\mathrm{CBF}$, which attained statistical significance at the $48 \mathrm{~h}$ time point. By $7 \mathrm{~d}$ after hypoxia-ischemia, when the animals were $2 \mathrm{wk}$ of age, there were no detectable left-right differences in the MRI maps for either gray or white 


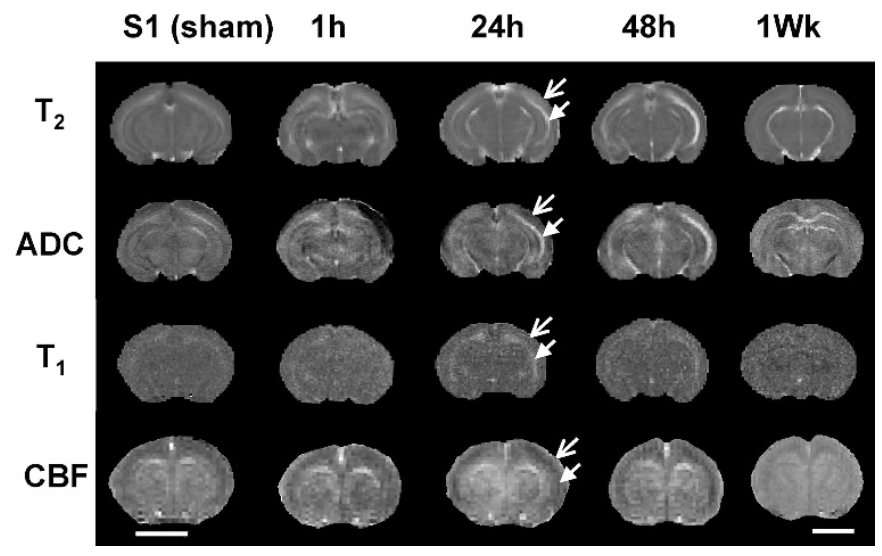

Figure 1. Representative MRI maps of $T_{2}, T_{1}$, the ADC of water and CBF from posterior cerebrum scans in a sham rat at $8 \mathrm{~d}$ of age (S1) or rats at different times following a rather mild hypoxic-ischemic insult. Selective changes within white matter (closed arrows) compared with cortical gray matter (open arrows). Younger brains are magnified slightly more than older brains, resulting in the 5 -mm scale bars varying between young and older rats as shown.

matter regions. Within anterior slices of the cerebrum, generally similar ADC and $\mathrm{T}_{2}$ changes were observed within the external capsule and parietal cortex and changes in the striatum were similar to those in the thalamus.

Histologic changes. Hematoxylin and eosin-stained sections from 14 animals without cortical infarcts revealed hypoxic-ischemic changes in white matter different from those in gray matter. Changes in the external capsule within the hypoxic-ischemic hemisphere ranged from tissue being spongiform and/or rarefied with pyknosis to small cystlike lesions with gliosis and/or atrophy in eight of 14 brains (Fig. $3 A$ and $B)$. Within the gray matter of the ipsilateral hemisphere in the parietal cortex, there was evidence of mild gliosis and neuronal vacuolar changes in three of 14 rats, with no apparent pyknosis or atrophy in these regions.

In sham brains of 2-wk-old rats, there was a mild level of GFAP immunoreactivity within the white matter of the external capsule, whereas GFAP immunoreactivity was essentially negative in the cortex, striatum, and thalamus (Fig. $3 C$ and $D$ ). In the hypoxic-ischemic brains of rats $1 \mathrm{wk}$ after hypoxiaischemia, the number of GFAP-positive immunoreactive astrocytes was increased to moderate or severe levels of staining in the ipsilateral external capsule in eight of 14 rats and staining was also mildly elevated in the ipsilateral parietal cortex, thalamus, or striatum of several (three to six) rats. Generally, GFAP staining in the contralateral hemisphere was comparable with that of sham controls.

In sections from sham animals at 2-wk of age, MBP immunoreactivity was observed in white matter tracts throughout the cerebrum. This immunoreactivity was intense in the external capsule (Fig. $3 E-G$ ) extending into the adjacent cortex without major left-right hemispheric differences in the sham brains. A relative decrease of MBP immunoreactivity was often observed ipsilaterally (e.g. in 10 of 14 animals) resulting in modest reductions in the ipsilateral/contralateral ratios of relative OD (e.g. $0.95 \pm 0.06$ and $0.99 \pm 0.09$ in posterior
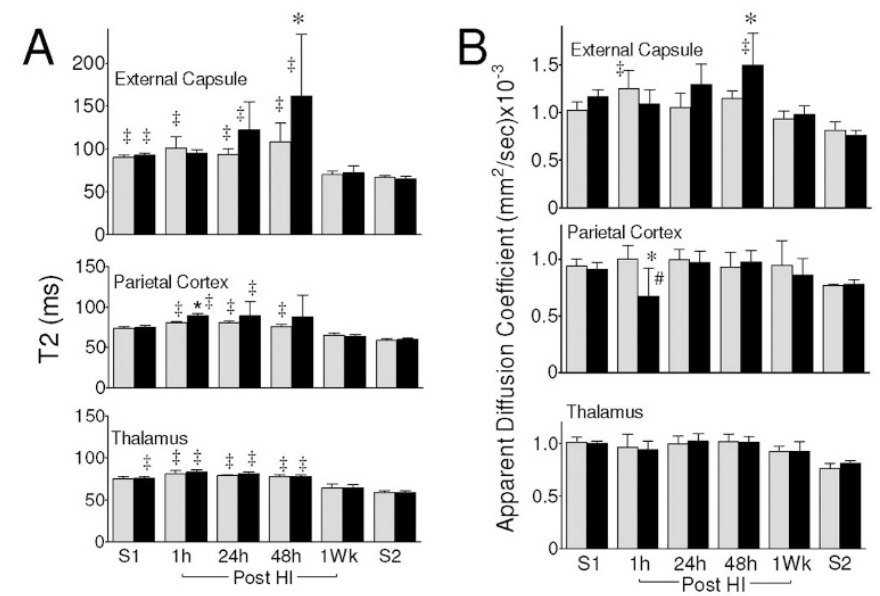

\section{C}
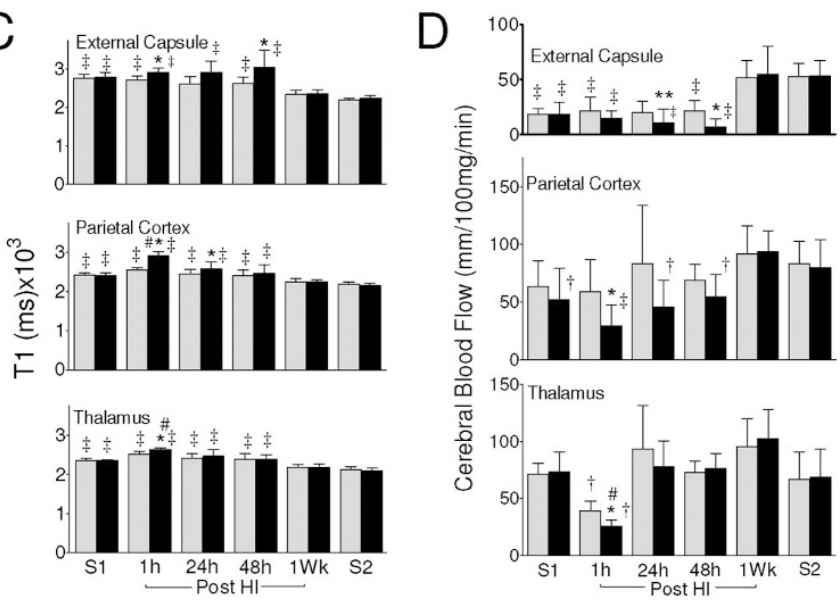

Figure 2. Quantitative changes in $\mathrm{T}_{2}(A), \mathrm{ADC}$ of water $(B), \mathrm{T}_{1}(C)$, and $\mathrm{CBF}$ $(D)$ in brain regions from posterior cerebrum following a mild cerebral hypoxic-ischemic insult in neonatal rats. Regions of interest included white matter (external capsule) and gray matter (parietal cortex and thalamus) ipsilateral (solid columns) or contralateral (shaded columns) to the carotid artery occlusion. Mean values are shown for 8-d-old $(S 1)$ or 2-wk-old (S2) sham animals and for times following hypoxia-ischemia of 1, 24, and $48 \mathrm{~h}$ and $1 \mathrm{wk}$ after hypoxia-ischemia $(H I)$. The number of artifact-free scans per group was four to six, with the exception of 14 in $1 \mathrm{Wk}$ groups $(A, C$, and $D)$ and two in S2 groups $(B) .{ }^{*} p<0.05 ; * * p<0.01$ different from contralateral hemisphere (paired $t$ test). $\dagger p<0.05$, different from $1 \mathrm{Wk}$ HI and S2 groups; $\ddagger p<0.05$, different from $1 \mathrm{Wk}$ HI group; \#p $<0.05$, different from $48 \mathrm{~h} \mathrm{HI}$ group (ANOVA and Bonferroni $t$ test).

external capsule of hypoxia-ischemia or sham animals, respectively; $p<0.05$ ).

In sham brains of 2-wk-old rats, there was normal or scant TUNEL labeling within the white matter of the external capsule, cortex, and striatum (Fig. 4). In the brains of hypoxicischemic rats, a moderate to severe grade of TUNEL-positive labeling was observed within the ipsilateral external capsule. There was also mild TUNEL labeling in the contralateral external capsule in the hypoxia-ischemia animals, but positive labeling was greater ipsilaterally in eight of 14 animals. In the parietal cortex and striatum, TUNEL labeling contralaterally was normal and ipsilaterally a mild to moderate increase was observed in only two to five hypoxic-ischemic brains.

Overall, the majority (eight of 14) of animals with substantial left-right differences in their MRI (e.g. $\left.\mathrm{T}_{2}\right)$ had one or more ipsilateral changes in their histologic staining. Three 


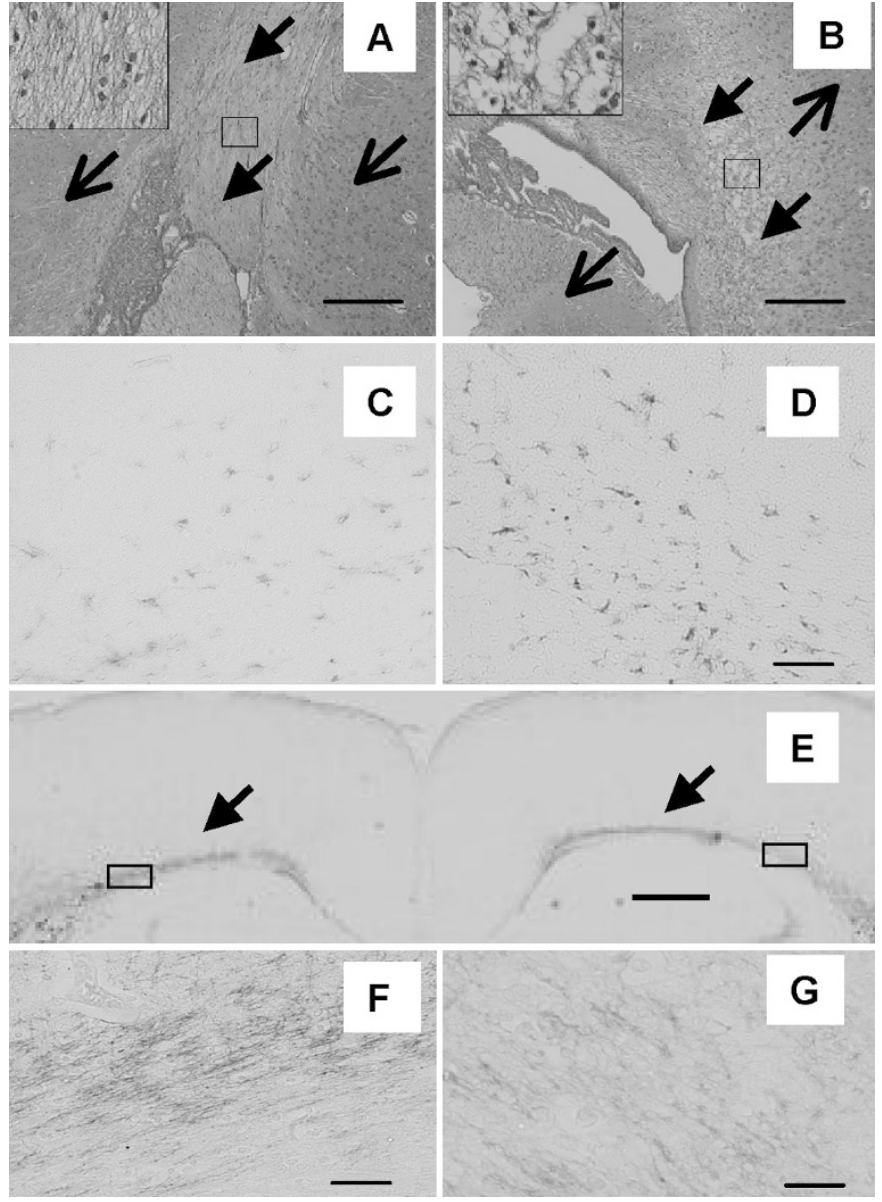

Figure 3. Representative micrographs containing white matter (closed arrows) and gray matter (open arrows) in sections stained with hematoxylin and eosin $(A, B)$, GFAP $(C, D)$ or MBP $(E-G)$. Sections are from the hemisphere either contralateral $(A, C, F)$ or ipsilateral $(B, D, G)$ to a mild hypoxicischemic episode $1 \mathrm{wk}$ after the insult. Staining in sham animals was similar to that in the contralateral hemisphere. Shown in the insets at higher magnification $(A, B)$ are signs of hypoxic-ischemic injury including spongiform or rarefaction changes of the white matter and the presence of some pyknotic cells. GFAP immunostaining shows hypertrophy and an increased number of reactive astrocytes ipsilateral to the injury $(D)$ compared with that contralaterally $(C)$. A decrease in MBP immunostaining was observed in the ipsilateral external capsule (e.g. boxes in $E$ ) and are shown magnified in $G$ and $F$. Scale bars indicate $250 \mu \mathrm{m}$ for $A$ and $B ; 100 \mu \mathrm{m}$ for $C, D, F, G$; and $1 \mathrm{~mm}$ for $E$.
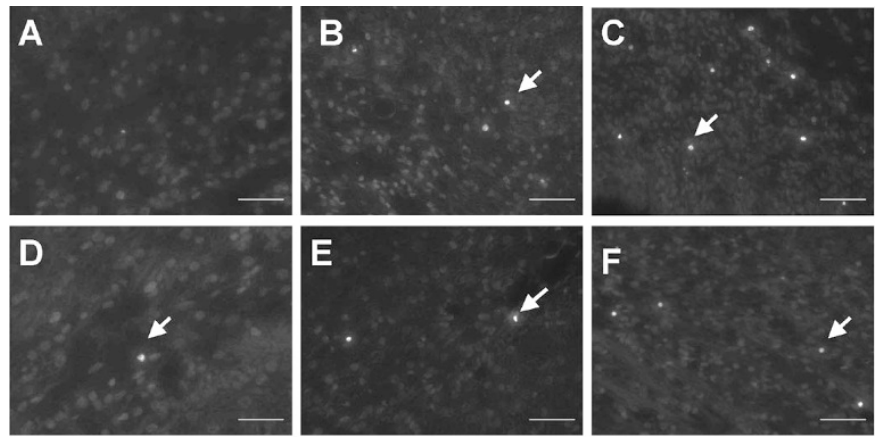

Figure 4. Representative micrographs of TUNEL-stained sections containing white matter within the cingulum $(A-C)$ and external capsule $(D-F) 1 \mathrm{wk}$ after a mild hypoxic-ischemic insult in 1-wk-old rats. TUNEL-positive cells ( $a r-$ rows) were increased in the hemisphere ipsilateral $(C, F)$ to the hypoxiaischemia compared with that in sham animals $(A, D)$ or contralaterally $(B, E)$ (scale bar $=50 \mu \mathrm{m}$ ). animals (one in the 1-h hypoxia-ischemia group) had histologic changes without MRI changes, whereas another three animals had neither MRI changes nor appreciable ipsilateral increases in GFAP or TUNEL labeling.

\section{DISCUSSION}

White matter injury with a hypoxic-ischemic pathogenesis is detected fairly frequently in human infants; however, the evolution of acute and subacute MRI changes are difficult to establish in these sick infants. The present study uses a rather mild model of hypoxic-ischemic injury to investigate the evolution of MRI changes following a relatively selective white matter injury in neonatal rats. The results demonstrate that a relatively mild cerebral hypoxic-ischemic insult (shorter hypoxia in duration and at a slightly lower temperature than that normally producing infarcts) produces in the majority of animals early MRI changes that initially are less pronounced in white than gray matter, but these changes enhance in white matter at 24-48 $\mathrm{h}$ after hypoxia-ischemia. Unusual was the delayed increase in ADC along with increases in $\mathrm{T}_{1}$ and $\mathrm{T}_{2}$ within white matter in the first $2 \mathrm{~d}$ following hypoxiaischemia, at which time MRI changes in gray matter had generally recovered. By $7 \mathrm{~d}$ after hypoxia-ischemia, there were no left-right hemispheric differences of $\mathrm{ADC}, \mathrm{T}_{1}$ or $\mathrm{T}_{2}$ in either white or gray matter, although histologic examination demonstrated a tissue injury in white matter at this time. The results also demonstrate a correspondence between the standard MRI changes and regional reductions in CBF at acute times following the insult.

Previous MRI studies of hypoxic-ischemic changes in immature animal models have focused predominantly on the evolution of MRI changes in gray matter following a moderately severe episode of hypoxia-ischemia (e.g. duration of hypoxia-ischemia of $1.5 \mathrm{~h})(12,15,16)$, resulting in cortical and subcortical infarction and similar gray/white matter MRI changes over the first $24 \mathrm{~h}$ (16). Studies in human infants have also usually investigated MRI changes following relatively severe brain injury, and there has been a focus on ADC because of the early changes in ADC known to occur with ischemia. Without distinguishing gray or white matter localization, McKinstry et al. (10) observed acute reductions in ADC within the first few days and pseudonormalization by 1 wk after birth. However, the severity of the insult likely affects the region affected, with white matter being more affected than gray following relatively mild cerebral hypoxia-ischemia or partial asphyxia $(11,17)$. A study of neonatal hypoxicischemic encephalopathy demonstrated that with more severely injured white matter, there were early reductions in ADC in the first few days following birth, a pseudonormalization at the end of $1 \mathrm{wk}$, whereas with moderate white matter lesions, ADC was within the normal range on early scans and tended to be increased after $7 \mathrm{~d}$ (8). The latter findings are consistent with the results of the current study, considering that hypoxic-ischemic tissue changes and their MRI correlates appear to evolve faster in animal models than those observed clinically $(10,18,19)$. Together these studies indicate that the evolution of MRI changes appears to depend on the severity of 
the hypoxic-ischemic insult and the region affected (e.g. white versus gray matter).

Early reductions in ADC, either during or after a moderately severe hypoxic-ischemic insult, have been reported in gray and white matter $(5-7,9,10)$ and are generally considered to correspond to cell swelling and reductions in extracellular space (18). ADC increases in white matter potentially associated with hypoxia-ischemia are reported less frequently and are not as well understood $(6,11,20)$. It is known there are developmental reductions in ADC (e.g. between 28 and 40 wk) believed to be related to developmental changes in preoligodendroglia and myelination contributing to decreases in extracellular space and water content in cerebral white matter (4). Thus, relative ADC increases observed in premature infants with a diffuse white matter abnormality were proposed to be related to poor white matter development following injury (6). However, it is also possible that vacuolation and fiber rarefaction within the white matter, as has been observed in our model of cerebral hypoxia-ischemia at $24 \mathrm{~h}$ after hypoxia-ischemia, can be a cause of the ADC increases presently (11). This would be similar to the increase in ADC observed following permanent middle cerebral artery occlusion in the rat being associated with edema and an enlargement of extracellular space between white matter fibers (21). It is also believed that the ADC increase may be associated with reduced cell volumes and increased extracellular space associated with apoptotic cell death $(8,22)$, and there is support for such cell death following hypoxia-ischemia in white matter $(16,23,24)$. Determination of the extent to which white matter edema and cell death or other tissue changes contribute to the ADC increases will require further study.

Unavailable routinely in the clinic are perfusion maps that were also acquired in the present study providing information on the potential contribution of blood flow changes to the injury. Previously, we measured CBF changes in cortex within $24 \mathrm{~h}$ following a moderately severe hypoxic-ischemic insult in neonatal rats and demonstrated a return of CBF in parietal cortex toward normal by $24 \mathrm{~h}$ after hypoxia-ischemia (25). In contrast, in the present study, CBF in the ipsilateral white matter tended to be reduced at $1 \mathrm{~h}$ after hypoxia-ischemia, decreasing further at 24 and $48 \mathrm{~h}$ after hypoxia-ischemia. However, because flow levels were only moderately reduced, they likely reflect a secondary energy failure or injury rather than contributing to the injury itself.

Despite evidence of relatively selective injury in white matter such as gliosis, cell death, reduced myelination, and some edematous changes present at $1 \mathrm{wk}$ after hypoxiaischemia, the MRI changes in all regions had normalized. This possibly could be explained by tissue injury being obscured by a reduced contrast between white and gray matter at $2 \mathrm{wk}$ of age associated with developmental changes in $\mathrm{T}_{1}, \mathrm{~T}_{2}$, and ADC $(4,26)$. Whether this is the case would need long-term MRI in this model, but one or more standard MRI sequences are unlikely to assess definitively the onset or existence of permanent damage. Indeed, the use of MRI for accurately determining white matter injury still needs a better understanding of the regional evolution of MRI changes for different severities of injury and possibly the addition of other sequences. Promising MRI methods that measure other aspects of the tissue such as altered metabolism or delayed myelination include methods such as proton spectroscopy, diffusion tensor imaging, and measures of fractional anisotropy or magnetization transfer $(7,27,28)$. However, such methods require lengthy acquisition times or are technically difficult in these small animals and were beyond the scope of the present study.

In conclusion, our present results indicate that when using standard imaging techniques such as $\mathrm{T}_{2}$ and $\mathrm{ADC}$ to diagnose a mild hypoxic-ischemic white matter insult, the optimal time for detecting ischemic injury will be in the first few days rather than hours after the insult, and this period may be extended in some patients. Increases in ADC at $24-48 \mathrm{~h}$ after hypoxia-ischemia provide relatively early signs of mild white matter injury likely reflecting at least in part a white matter increase in water content and extracellular space. At subacute times, these MRI sequences do not necessarily detect tissue changes that can include reduced myelination, cell death, or astrocytic proliferation.

Acknowledgments. The authors thank N. Klementis, H. Meghani, L. Lin, and D. Rushforth for excellent technical assistance and T. Foniok for expert acquisition of the magnetic resonance images.

\section{REFERENCES}

1. Volpe JJ 2001 Neurobiology of periventricular leukomalacia in the premature infant. Pediatr Res 50:553-562

2. Back SA 2001 Recent advances in human perinatal white matter injury. Prog Brain Res 132:131-147

3. Volpe JJ 2003 Cerebral white matter injury of the premature infant-more common than you think. Pediatrics 112:176-180

4. Huppi PS 2002 Advances in postnatal neuroimaging relevance to pathogenesis and treatment of brain injury. Clin Perinatol 29:827-856

5. Bozzao A, Di Paolo, A, Mazzoleni C, Fasoli F, Simonetti A, Fantozzi LM, Floris R 2003 Diffusion-weighted MR imaging in the early diagnosis of periventricular leukomalacia. Eur Radiol 13:1571-1576

6. Counsell SJ, Allsop JM, Harrison MC, Larkman DJ, Kennea NL, Kapellou O, Cowan FM, Hajnal JV, Edwards AD, Rutherford MA 2003 Diffusion-weighted imaging of the brain in preterm infants with focal and diffuse white matter abnormality. Pediatrics 112:1-7

7. van Pul C, Buijs J, Janssen MJ, Roos GF, Vlaardingerbroek MT, Wijn PF 2005 Selecting the best index for following the temporal evolution of apparent diffusion coefficient and diffusion anisotropy after hypoxic-ischemic white matter injury in neonates. AJNR Am J Neuroradiol 26:469-481

8. Rutherford M, Counsell S, Allsop J, Boardman J, Kapellou O, Larkman D, Hajnal J, Edwards D, Cowan F 2004 Diffusion-weighted magnetic resonance imaging in term perinatal brain injury: a comparison with site of lesion and time from birth. Pediatrics 114:1004-1014

9. Inder T, Huppi PS, Zientara GP, Maier SE, Jolesz FA, di Salvo, D, Robertson R, Barnes PD, Volpe JJ 1999 Early detection of periventricular leukomalacia by diffusion-weighted magnetic resonance imaging techniques. J Pediatr 134:631-634

10. McKinstry RC, Miller JH, Snyder AZ, Mathur A, Schefft GL, Almli CR, Shimony JS, Shiran SI, Neil JJ 2002 A prospective, longitudinal diffusion tensor imaging study of brain injury in newborns. Neurology 59:824-833

11. Qiao M, Meng S, Scobie T, Foniok T, Tuor UI 2004 Magnetic resonance imaging of differential gray versus white matter injury following a mild or moderate hypoxic-ischemic insult in neonatal rats. Neurosci Lett 368:332-336

12. Tuor UI, Kozlowski P, Del Bigio, MR, Ramjiawan B, Su S, Malisza K, Saunders JK 1998 Diffusion- and T2-weighted increases in magnetic resonance images of immature brain during hypoxia-ischemia: transient reversal posthypoxia. Exp Neurol $150: 321-328$

13. Knight RA, Dereski MO, Helpern JA, Ordidge RJ, Chopp M 1994 Magnetic resonance imaging assessment of evolving focal cerebral ischemia. Comparison with histopathology in rats. Stroke 25:1252-1261

14. Lei H, Peeling J 1999 A strategy to optimize the signal-to-noise ratio in one-coil arterial spin tagging perfusion imaging. Magn Reson Med 41:563-568

15. Rumpel H, Nedelcu J, Aguzzi A, Martin E 1997 Late glial swelling after acute cerebral hypoxia-ischemia in the neonatal rat: a combined magnetic resonance and histochemical study. Pediatr Res 42:54-59 
16. Meng S, Qiao M, Foniok T, Tuor UI 2005 White matter damage precedes that in gray matter despite similar magnetic resonance imaging changes following cerebral hypoxia-ischemia in neonatal rats. Exp Brain Res 166:56-60

17. Miller SP, Ramaswamy V, Michelson D, Barkovich AJ, Holshouser B, Wycliffe N, Glidden DV, Deming D, Partridge JC, Wu YW, Ashwal S, Ferriero DM 2005 Patterns of brain injury in term neonatal encephalopathy. J Pediatr 146:453-460

18. Qiao M, Malisza KL, Del Bigio, MR, Tuor UI 2002 Transient hypoxia-ischemia in rats: changes in diffusion-sensitive MR imaging findings, extracellular space, and $\mathrm{Na}+-\mathrm{K}+$-adenosine triphosphatase and cytochrome oxidase activity. Radiology 223:65-75

19. Li F, Liu KF, Silva MD, Meng X, Gerriets T, Helmer KG, Fenstermacher JD, Sotak $\mathrm{CH}$, Fisher M 2002 Acute postischemic renormalization of the apparent diffusion coefficient of water is not associated with reversal of astrocytic swelling and neuronal shrinkage in rats. AJNR Am J Neuroradiol 23:180-188

20. Baud O, Daire JL, Dalmaz Y, Fontaine RH, Krueger RC, Sebag G, Evrard P, Gressens P, Verney C 2004 Gestational hypoxia induces white matter damage in neonatal rats: a new model of periventricular leukomalacia. Brain Pathol 14:1-10

21. Pierpaoli C, Righini A, Linfante I, Tao-Cheng JH, Alger JR, Di Chiro, G 1993 Histopathologic correlates of abnormal water diffusion in cerebral ischemia: diffusion-weighted MR imaging and light and electron microscopic study. Radiology 189:439-448
22. Brauer M 2003 In vivo monitoring of apoptosis. Prog Neuropsychopharmacol Biol Psychiatry 27:323-331

23. Back SA, Han BH, Luo NL, Chricton CA, Xanthoudakis S, Tam J, Arvin KL, Holtzman DM 2002 Selective vulnerability of late oligodendrocyte progenitors to hypoxia-ischemia. J Neurosci 22:455-463

24. Skoff RP, Bessert DA, Barks JD, Song D, Cerghet M, Silverstein FS 2001 Hypoxicischemic injury results in acute disruption of myelin gene expression and death of oligodendroglial precursors in neonatal mice. Int J Dev Neurosci 19:197-208

25. Qiao M, Latta P, Foniok T, Buist R, Meng S, Tomanek B, Tuor UI 2004 Cerebral blood flow response to a hypoxia-ischemia insult differs in neonatal and juvenile rats. MAGMA 17:117-124

26. Barkovich AJ 2000 Concepts of myelin and myelination in neuroradiology. AJNR Am J Neuroradiol 21:1099-1109

27. Partridge SC, Mukherjee P, Henry RG, Miller SP, Berman JI, Jin H, Lu Y, Glenn OA, Ferriero DM, Barkovich AJ, Vigneron DB 2004 Diffusion tensor imaging: serial quantitation of white matter tract maturity in premature newborns. Neuroimage 22:1302-1314

28. Miller SP, Newton N, Ferriero DM, Partridge JC, Glidden DV, Barnwell A, Chuan NA, Vigneron DB, Barkovich AJ 2002 Predictors of 30-month outcome after perinatal depression: role of proton MRS and socioeconomic factors. Pediatr Res 52:71-77 